ISSN: 2637-4692

\title{
Implant Treatment Planning: A Time to Remember your Physics
}

\section{Charles D Schlesinger*}

Private practice Albuquerque, USA

*Corresponding author: Charles D Schlesinger, FICOI, Private practice Albuquerque, USA

\section{Case Report}

When treatment planning for implant treatment is imperative that we not only assess the dental condition that requires treatment, but also the physics that will be involved in the case. Whether this is a single tooth or a full arch, the success long term relies heavily on making sure the forces that the implant and the peri-implant tissues are controlled. One major key to success is placing the implant in the best prosthetically guided position- never compromise this key point, it is critical that esthetic concerns do not outweigh factors used to promote successful osseointegration [1]. Implants are not teeth and should never be treated the same way (Figure 1). Without the advantages afforded by a pdl, the "ankylosed" implant cannot accommodate forces the same way as natural teeth. The bone surrounding the implant take the brunt of the forces that are absorbed by the pdl surrounding a natural tooth.

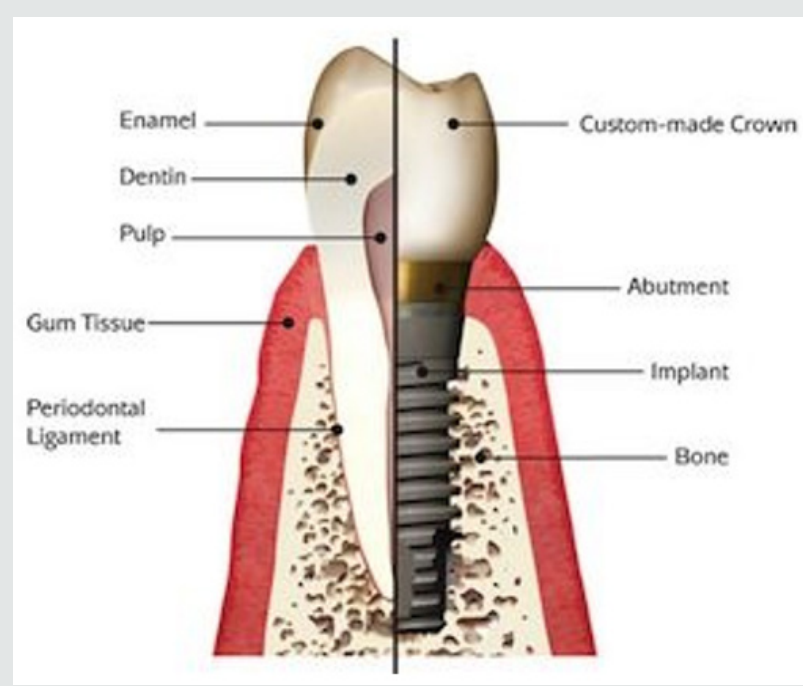

Figure 1: The difference between a tooth and implant.

The first requirement for successful osseointegration is primary stability. At the time of placement this is determined by the final seating torque, or more accurately, through RFA with an Osstell or Penguin device (Figure 2 \& 3). By having enough stability to resist 150 microns of movement, integration will occur [2]. When comparing the accuracy of stability measurements [3], even though seating torque is a great predictor of stability and is backed up both anecdotal and research evidence, RFA is considered more accurate in the literature consensus.

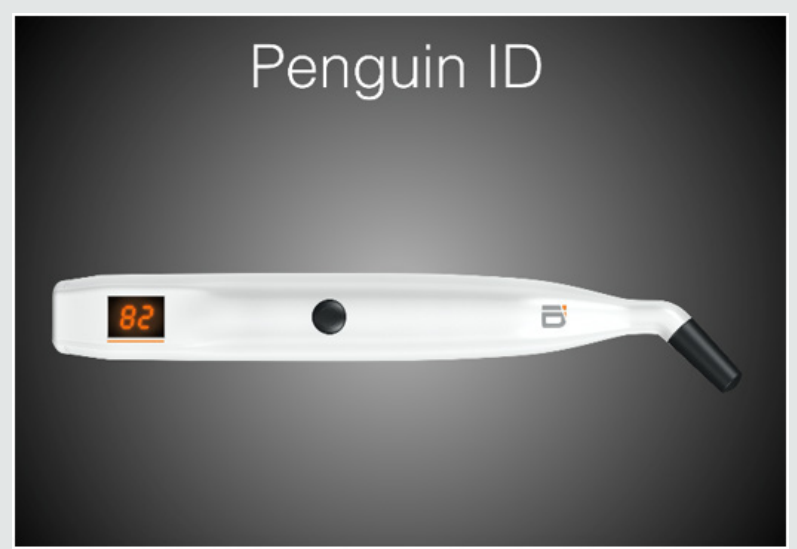

Figure 2: The Penguin RFA device.

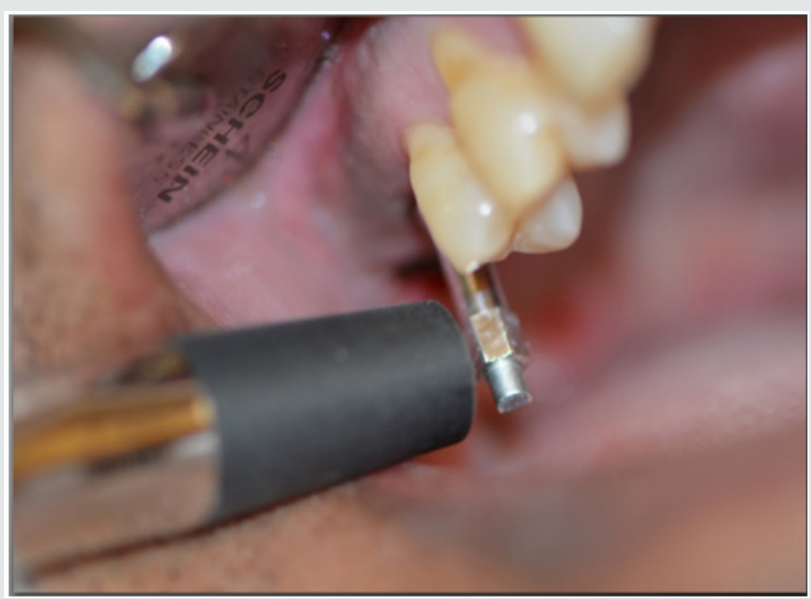

Figure 3: Using RFA to clinically determine implant stability. 
We can decrease the forces to the peri-implant bone through the following means

I. Increase surface area

a) Implant \# or size

II. Decrease force on implants

b) Patient factors, occlusal load direction, implant position

III. Mechanical properties of bone

c) Cortical vs trabecular

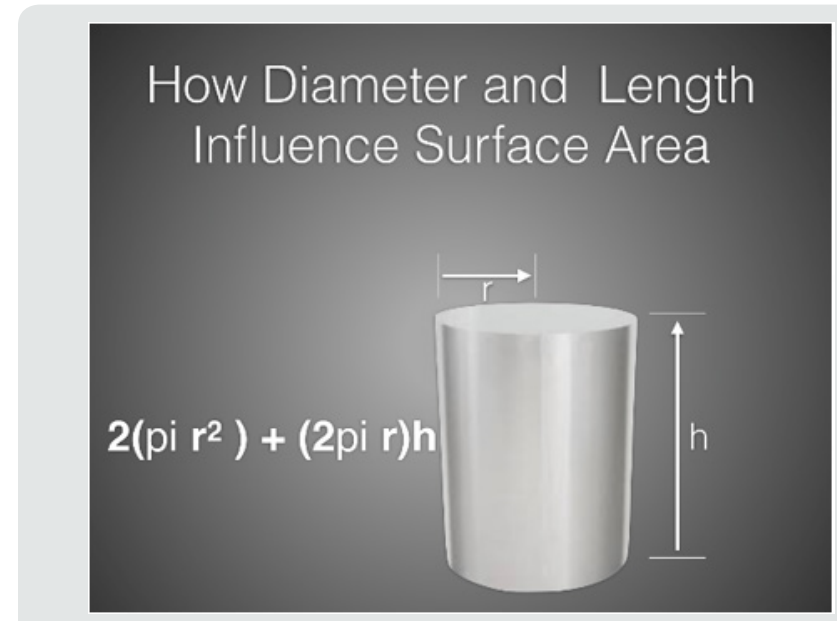

Figure 4: Calculating the surface if a cylinder (implant).

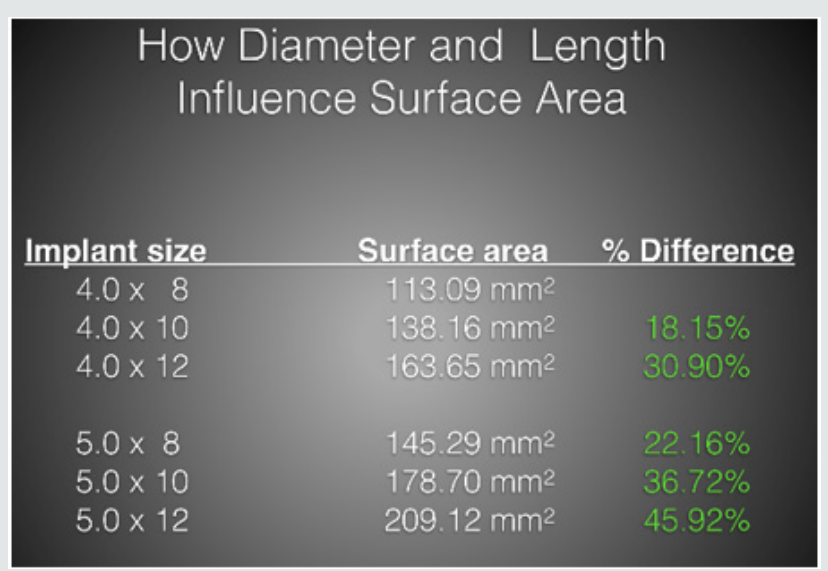

Figure 5: How length and width of an implant affect forces.

By dispersing the masticatory forces over multiple implants, the individual load on each implant can be reduced. This happens in situations where multiple implants are tied together by a single restoration such as a bridge or a full arch restoration. In this type of situation, each implant shares the total load and forces generated to the hard tissue is minimized. In both multi and single unit cases, the forces to the per-implant bone can also be decreased by a larger surface area of bone-to-implant contact. Pressure caused by the implant laterally loading the bone around it will cause bone to resorb. If we look at the formula for pressure; $\mathrm{P}=$ Force/area, we can see that if we increase the area of implant to bone contact, the overall pressure will be decreased to any particular area. This increase in surface area can be achieved by either increasing the length of the implant or increasing the diameter or both (Figure 4 \&5).

The next important part of the equation is the patient- As patients age, their masticatory forces decrease. The forces females produce are generally lower than those produced by men and tend to decrease earlier in life than those of their male counterparts. Women tend to have a decrease after 25 years of age, while males decrease after 45 years [4]. The more dolichofacial they are, the lower the force generated. Brachyfacial individuals have shorter and thicker masseters which produce high forces with a more efficient lever system (Figure 6).

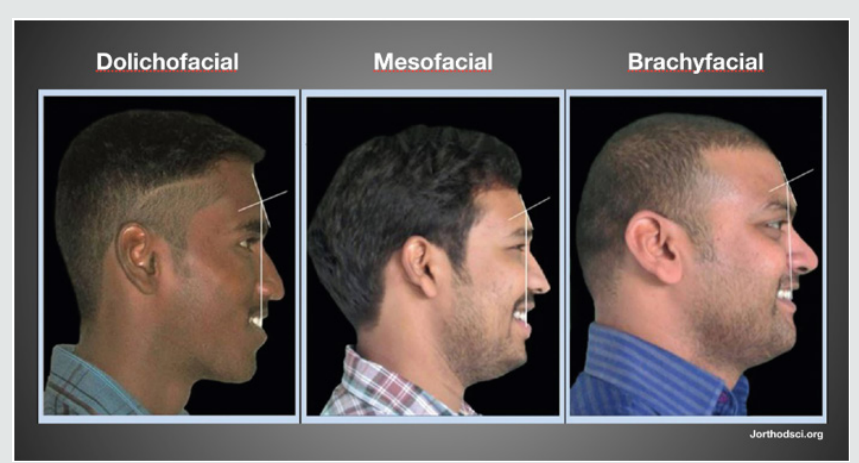

Figure 6: Facial profiles.

The position of the restoration plays a role in the potential forces generated along with the type of opposing dentition. The closer the restoration is to the TMJ, the higher the forces (Figure 7). This is like the force vs position when placing a nut in a nutcrackerthe closer to the hinge, the easier it is to break the nut.

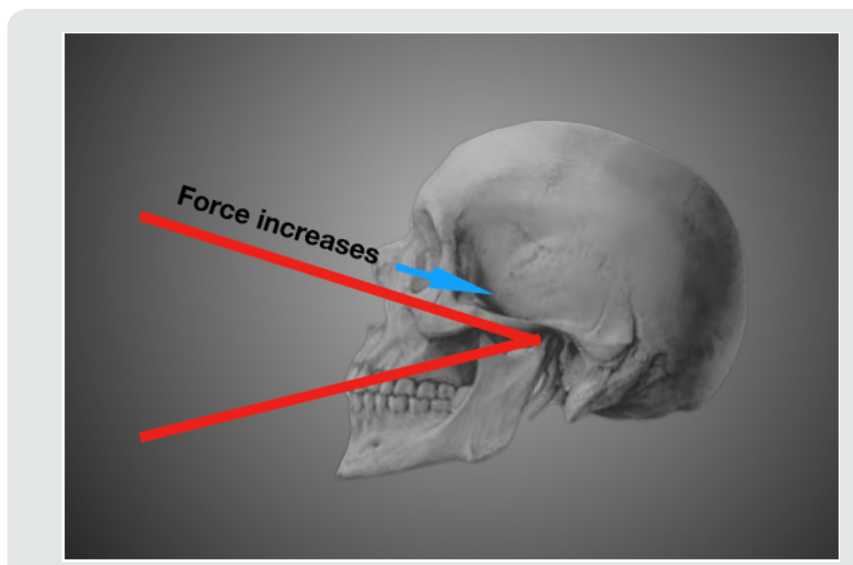

Figure 7: The "nutcracker" principle.

Evaluating what type of opposing dentition contributed greatly to the amount of force an implant and its restoration will receive. Natural dentitions can produce 250psi or greater while a full denture produce as low as $25 \mathrm{psi}$. On top of these factors, those patients who suffer from bruxism can have masseter strength $6 x^{\prime} \mathrm{s}$ that of a non-bruxer [5].

Another important factor to consider when placing implants is the direction of the force to be exerted on it. Both implant components and peri-implant tissues accept axial loading much better than off-axis forces. In the posterior, axial loading should 
always be the goal unless it is part of a multi-unit system. In the anterior, off-axis loading is many times unavoidable, but the forces are lower than the posterior and therefore not as much of an issue.

A cantilevering force can be produced when there is a large amount of restoration that extends from the implant body to the adjacent tooth. The larger this cantilever, the more psi is exerted on the restoration and implant components (Figure 8). A vertical cantilevering force can be produced when an implant restoration becomes excessively "tall". In general, the restoration-to-implant length ration should not exceed 2.5: 1 .

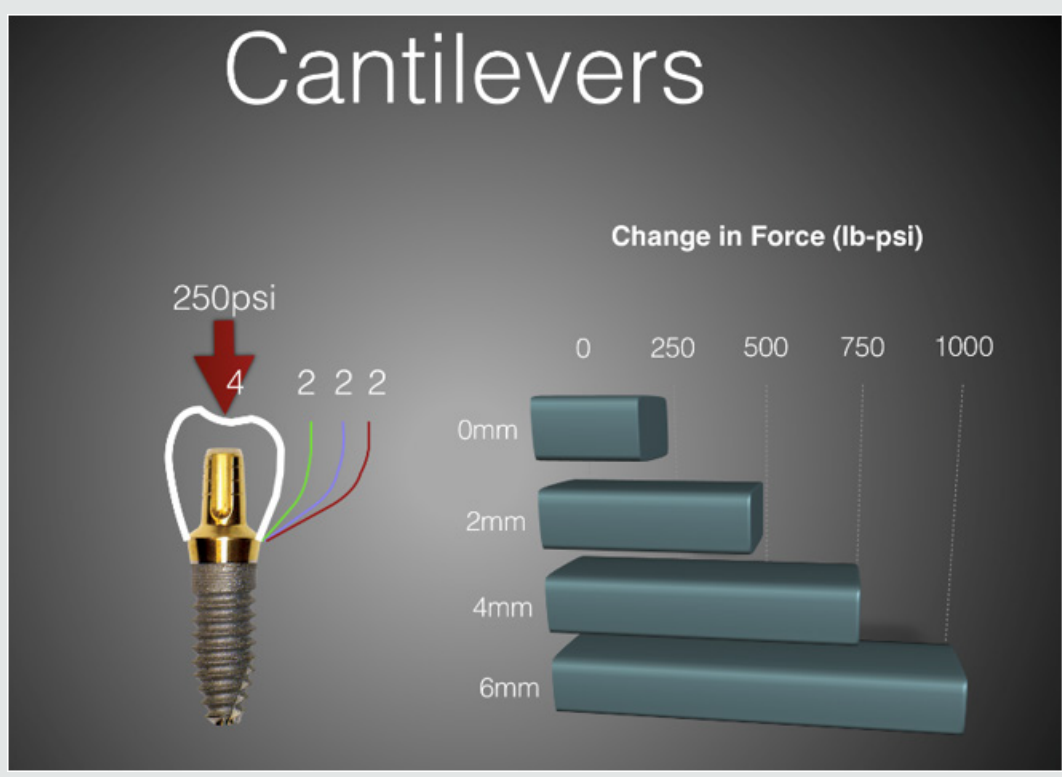

Figure 8: How cantilevers increase loads.

The final component to success is the quality of bone the implant is placed in. As we know bone is classified from D1, made up mostly of cortical bone to D4 which is very soft and is primarily trabecular in make-up (Figure 9). As clinicians this is an important factor to consider when choosing implant diameter and length.

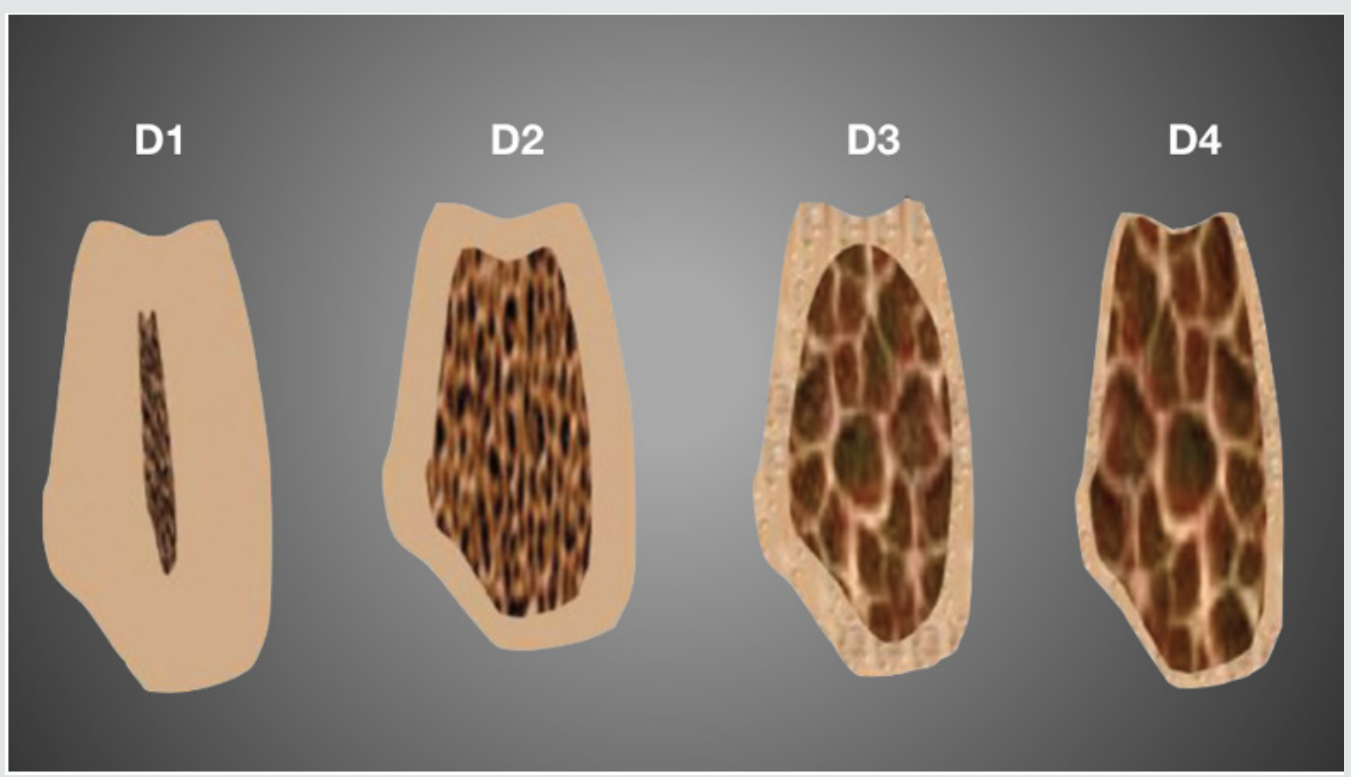

Figure 9: Bone types.

ISQ values for implant placement in D1 bone were significantly higher than those for implants placed in D3 bone. In D1 bone, the implant length does not make any significant difference in primary stability and in D3 bone, the primary stability of the implant increased when longer implants were utilized [6].
Restoring the human dentition is a complicated task due to the varied factors that contribute to success or failure in both the shortand long-term treatment. By assessing the whole patient and not just focusing on whether a tooth is missing or not, we can provide the very best treatment for our patients. 


\section{References}

1. Buser D, Martin W, Belser UC (2004) Optimizing esthetics for implant restorations in the anterior maxilla: anatomic and surgical considerations. Int J Oral Maxillofac Implants 19 Suppl: 43-61.

2. Schlesinger C (2019) How to confidently reduce treatment time. when to load an implant. The guide to monitoring implant stability, Osstell $\mathrm{AB}$ Sweden, pp. 40-42.

3. Rizkallah N, Fischer S, Kraut R (2013) Correlation between insertion torque and survival rates in immediately loaded implants in the maxilla: a retrospective study. Implant Dentistry 22(3): 250-254.
4. Shala K, Tmava-Dragusha A, Dula L, Pustina-Krasniqi T, Bicaj T, et al. (2018) Evaluation of maximum bite force in patients with complete dentures. Maced J sci 6(3): 559-563.

5. Duygu K, Arife D, Bek b (2010) Bite force and influential factors on bite force measurements: a literature review. Eur J Dent 4(2): 223-232.

6. Barikani H, Rashtak S, Akbari S, Badri S, Daneshparvar N, et al. (2013) The effect of implant length and diameter on the primary stability in different bone types. A J Dent (Tehran) 10(5): 449-455.
To Submit Your Article Click Here:

This work is licensed under Creative Commons Attribution 4.0 License

DOI: 10.32474/MADOHC.2020.04.000186

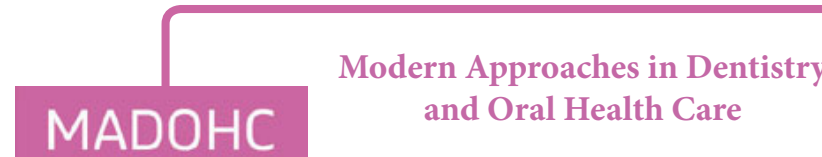

Assets of Publishing with us

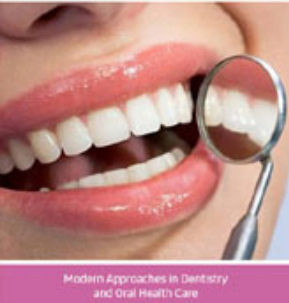

- Global archiving of articles

- Immediate, unrestricted online access

- Rigorous Peer Review Process

- Authors Retain Copyrights

- Unique DOI for all articles 